\title{
Optical Impulse Response of Semiconductor Optical Amplifiers in a Counter-Propagation Mach-Zehnder Switch
}

\author{
Deyu Zhou, Ivan Glesk, and Paul R. Prucnal \\ Department of Electrical Engineering \\ Princeton University , Princeton, NJ 08544 \\ Telephone: 609-258-2041, Fax: 609-258-2158 \\ Email:deyuzhou@princeton.edu
}

\begin{abstract}
Counter propagating control and signal pulse interactions exist in many important optical components in fiber optical communication networks and ultra-fast signal processing systems employing semiconductor optical amplifiers. Challenges facing this particular interaction configuration are considered analytically. Signal pulse propagation is studied by solving its traveling-wave equation under a perturbation approximation with the control pulse arriving at the opposite side of the semiconductor optical amplifier. The derived analytical expressions of optical impulse response clearly elucidate the physics involved in counter-propagating configurations.
\end{abstract}

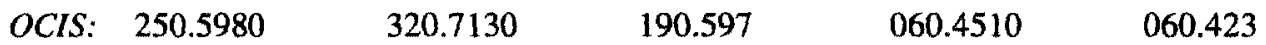

Semiconductor optical amplifiers (SOAs) are utilized in many important components such as demultiplexiers $[1,2]$, clock extractors $[3,4]$, wavelength converters $[5,6,7]$, packet routers [7], format converters [8], switches and logic gates [2,9] in optical time division multiplexed (OTDM), wavelength division multiplexed (WDM) communication networks and ultrafast optical signal processing systems. Many applications employ the SOAs with control and signal pulses counter-propagating with respect to each other. In terahertz optical asymmetric demultiplexier (TOAD) of OTDM applications as indicated in Fig. 1 [1,2], for example, the two SOAs in upper and lower arms of a Mach-Zehnder interferometer are displaced slightly off with respect to each other. An input data signal is split into two parts which are counter propagating with respect to a control pulse that demultiplexs the input signal. The switching window is determined by the small displacement $\Delta x$, the length of the SOA, and the optical impulse responses of the SOA. Wavelength converters ensure dynamic routing and switching in WDM networks. The counter-propagating input signals are converted into a different wavelength in a Mach-Zehnder wavelength converter [5,6]. In nonlinear interferometer (UNI) all-optical logic gates [9], the counter-propagating configuration of the control and signal pulse allows the logic gates to be cascadable. The control and signal pulse can be easily separated in devices that employ counter-propagating configurations, avoiding cross-talk and allowing the devices to cascade. The optical impulse response of the SOAs under counter propagating signal and control pulses is important to understand the performance of the devices. 


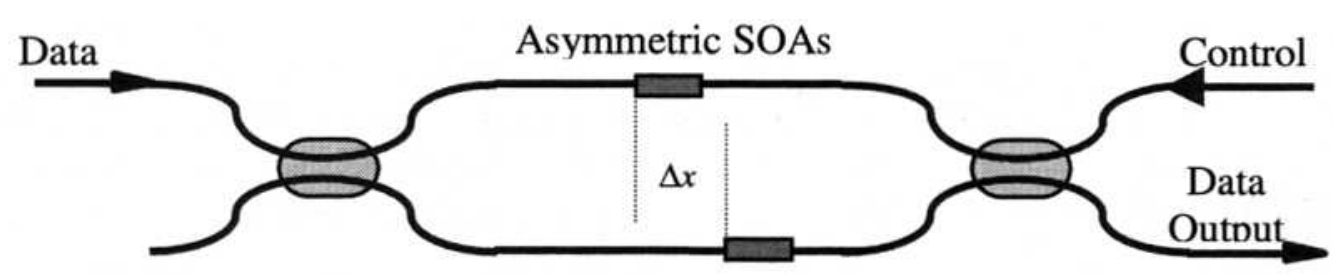

Fig. 1. A schematic diagram of a counter-propagating MZ TOAD switch. The switching window is determined by the displacement $\Delta x$, the length of the SOAs, and the optical impulse responses of the SOAs under counter-propagation configurations.

Theoretical studies by Mark and Mecozzi [10] and experimental work by Hall, et al. [11] have investigated the optical impulse response of active waveguides such as SOAs using a co-propagation configuration. Counter-propagating pump-and-probe experiments on an SOA have been conducted by Kang, et al. providing information on both nonlinear phase and gain dynamics [12]. Their experiments concentrated on the longer time scale of carrier recovery dynamics, on the order of several hundred picoseconds. Carrier recovery time, differential gain, saturation energy and the linewidth enhancement factor of the SOA were measured simultaneously in these pump-and-probe experiments [13]. However, the fast nonlinear dynamics due to carrier heating $(\mathrm{CH})$, two-photon absorption (TPA), spectral hole burning (SHB) and other fast nonlinear dynamic processes were not thoroughly investigated because of limited time resolution. In an attempt to analyze the TOAD switching window, an optical impulse response of the SOA has been proposed by Kang, et al., based on simple physical intuitions [14]. With this simple model, some of the experimental observations on TOAD switching windows can not be explained [15]. A more comprehensive model is needed. Up to now, a systematic theoretical analysis of the optical impulse response under counter-propagating geometry has not been studied.

There are three different features or challenges in the counter-propagating configurations. First, the time delay between control and signal pulses changes as pulses propagate inside the SOA. Second, the location where the control and signal pulses meet depends on the initial time delay, defined as the arrival-time difference at the entrance of the SOA. Third, the boundary conditions of this geometry are different from copropagating configurations. The control and signal pulses arrive at the SOA at opposite ends. However, for a fixed initial time delay and a fixed signal pulse location, the SOA response functions of the different nonlinear processes are essentially same as that of a co-propagating configuration, because typical SOAs are made of zinc blende semiconductors having central symmetry [16]. The third order nonlinear susceptibility, from which the material response functions stem, is invariant when the control is flipped into a counter propagating direction with respect to signal signal. In this paper, we use the medium response functions given in reference [10] from semi-classical density matrix theory for cases of fixed initial time delays and signal pulse locations. We then solve the travelling wave equations while taking care of the three above-mentioned challenges. An analytic optical impulse response is obtained for counter-propagating pump-and-probe type configurations. 
To avoid coherent artifacts $[11,17]$, we consider only the orthogonal control (pump) and signal (probe) pulses. The theory presented can be extended to parallel control and signal polarization as long as coherent interference is considered [11]. Since the control and signal pulses counter propagate with respect to each other as illustrated in Fig. 2, the time delay between control and signal pulses depends on the location $z$. If we assume that the control and signal pulses arrive at the zero side of an SOA of length $L$ at time $t_{p o}$ and $t_{s o}$, respectively, the initial time delay is $\tau_{o}=t_{s o}-t_{p o}$. Because the control pulse takes less time $z / v_{p}$ while the signal pulse takes more time $z / v_{s}$ to arrive at the location $z$, the time delay at the location $\tau_{z}$ is:

$$
\tau_{z}=t_{s o}+\frac{z}{v_{s}}-\left(t_{p o}-\frac{z}{v_{p}}\right)=\tau_{o}+\frac{z}{v_{p}}+\frac{z}{v_{s}} .
$$

Here, $v$ stands for group velocity and subscripts $p$ and $s$ denote for control and signal signals, respectively.

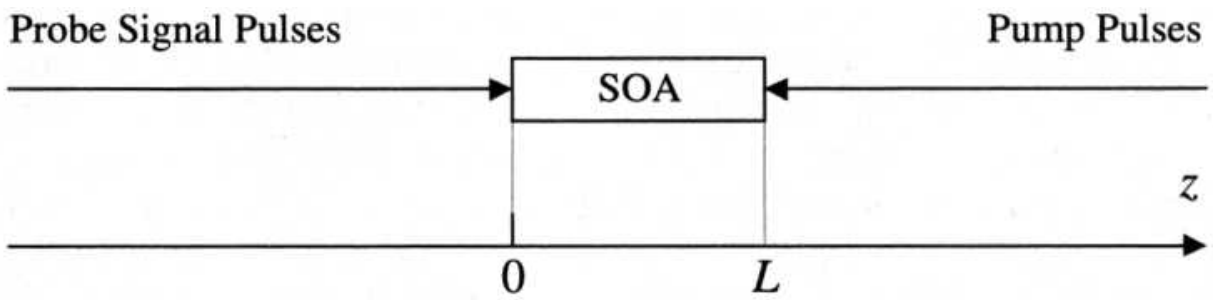

Arrival time at $z=0$

Pump pulse: $t_{p o}$ Probe pulse: $t_{s o}$

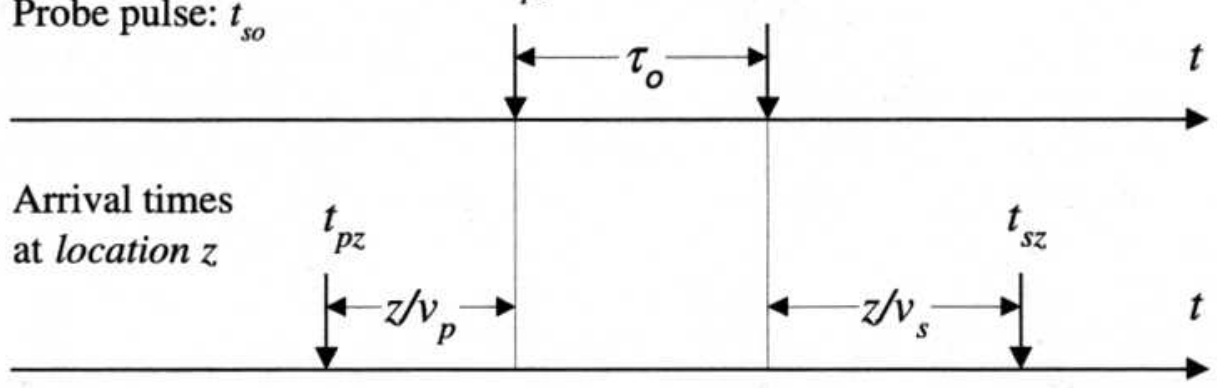

Fig. 2. A schematic diagram of boundary conditions and a timing diagram illustrating relationship between the time delay $\tau_{z}$ at the location $z$ and the initial time delay $\tau_{o}$. Here $t_{p o}, t_{p z}, t_{s o}$, and $t_{s z}$ are the arrival times of the control and signal pulses at locations 0 and $\mathrm{z}$, respectively. 
Control and signal pulses are normally derived from the same laser having same temporal shape and variable time delay. The control pulse energy is typically orders of magnitude larger than that of the signal pulse $[11,12]$. Contribution of the signal pulse to nonlinear polarization is negligible. Therefore, the travelling wave equation of the total field, which is summation of control and signal fields, is separable. Introducing slowly varying envelopes for control $E_{p}$ and signal $E_{s}$ pulses [10], noting that the control and signal pulses are orthogonal in polarization and propagate in opposite directions, we obtain the wave equations:

$$
\begin{gathered}
-\frac{\partial E_{p}(z, t)}{\partial z}=\hat{L}_{p} E_{p}(z, t)+\hat{R}_{p}(z, t) ; \\
\frac{\partial E_{s}(z, t)}{\partial z}=\hat{L}_{s} E_{s}(z, t)+\hat{R}_{s}(z, t) .
\end{gathered}
$$

Here, the linear operators $\hat{L}_{p}, \hat{L}_{s}$ and nonlinear operators $\hat{R}_{p}, \hat{R}_{s}$ are:

$$
\begin{aligned}
& \hat{L}_{j}=\frac{1}{2} \Gamma g_{j}\left(\omega_{o}+i \cdot \frac{\partial}{\partial t}\right)-\alpha_{\text {int }}+i \kappa_{j}\left(\omega_{o}+i \cdot \frac{\partial}{\partial t}\right) \equiv \frac{1}{2} \xi_{j}+i \hat{\kappa}_{j} \quad j=p, s \quad ; \\
& \hat{R}_{p}(z, t)=\int_{-\infty}^{\infty} h\left(t-t^{\prime}\right) \cdot\left|E_{p}\left(z, t^{\prime}\right)\right|^{2} \cdot E_{p}(z, t) \cdot d t^{\prime} ; \\
& \hat{R}_{s}(z, t)=\int_{-\infty}^{\infty} h\left(t+\tau_{z}-t^{\prime}\right) \cdot\left|E_{p}\left(z, t^{\prime}\right)\right|^{2} \cdot E_{s}(z, t) \cdot d t^{\prime} .
\end{aligned}
$$

Here, $i=\sqrt{-1}$. The optical confinement factor $\Gamma$ is used to account for the incomplete overlap of the transverse optical field distribution and the active region of the SOA. The parameters $g, \alpha_{\text {int }}$ and $\kappa$ describe the gain, intrinsic absorption and wavenumber change near the vicinity of the center frequency $\omega_{o}$ of the control and signal pulses. Here $h(t)$ is the material response of the SOA containing contributions from CH, TPA, SHB and other processes. Under semiclassical density matrix description, the analytic expression of $h(t)$ is given in reference [10].

To achieve a higher order of accuracy, i.e. to consider the effects of the control pulse saturation and self-phase modulation on the signal pulse, we have to consider the nonlinear term. In addition to the non-degenerated TPA considered in reference [10], the degenerated TPA becomes important in the material response $h(t)$ for control pulse propagation. In the first order perturbation approximation to which we consider, it is sufficient to consider the control pulse propagation to the zero order, completely neglecting the nonlinear term in Eq. (2a). The equation is solved under the boundary condition that control pulse enters the SOA from the right side of the SOA with a field distribution $E_{p}(L, t)$ as illustrated in Fig. 2:

$$
E_{p}(z, t)=e^{(L-z) \hat{L}_{p}} E_{p}(L, t) .
$$


When the signal field is expanded into the summation of two fields $E_{s o}(z, t)$---the signal field in absence of control pulse and $\Delta E_{s}(z, t)$---perturbation of the signal pulse under the influence of the control pulse, the solution for field $E_{s o}(z, t)$ is easily obtained as follows:

$$
E_{s o}(z, t)=e^{z \hat{L}_{s}} E_{s}(0, t)
$$

Here, $E_{s}(0, t)$ is the input signal field at the beginning of the SOA. $\Delta E_{s}(z, t)$ satisfies:

$$
\frac{\partial \Delta E_{s}(z, t)}{\partial z}=\hat{L}_{s} \Delta E_{s}(z, t)+\int_{-\infty}^{\infty} h\left(t+\tau_{z}-t^{\prime}\right) \cdot\left|E_{p}\left(z, t^{\prime}\right)\right|^{2} \cdot E_{s o}(z, t) \cdot d t^{\prime}
$$

The general solution is obtained by integration:

$$
\begin{aligned}
& \Delta E_{s}(z, t)=\int_{z_{o}}^{z} d z^{\prime} \cdot\left[e^{\left(z-z^{\prime}\right) \hat{L}_{s}} \int_{-\infty}^{\infty} h\left(t+\tau_{z}-t^{\prime}\right) \cdot\left|E_{p}\left(z, t^{\prime}\right)\right|^{2} \cdot E_{s o}(z, t) \cdot d t^{\prime}\right] \\
& z_{o}= \begin{cases}0 & \text { if } z 0<0, \\
z 0 & \text { if } z 0 \in[0, L], \\
L & \text { if } z 0>L .\end{cases}
\end{aligned}
$$

The lower bound $z_{o}$ of the integral reflects the three possible regimes of the location $z 0=-\frac{v_{p} v_{s}}{v_{p}+v_{s}} \cdot \tau_{p}$ where control and signal pulses meet for a given initial time delay $\tau_{o}$.

The output of the detector monitoring the changes in signal pulse due to the influence of control pulse is expressed as:

$$
I\left(\tau_{o}\right)=\int_{-\infty}^{\infty} d t \cdot E_{s o}^{*}(L, t) \cdot \Delta E_{s}(L, t)
$$

Here, the symbol * stands for a complex conjugate. The output contains both amplitude and phase changes which are contained in its real and imaginary parts, respectively. The real part is detected by the measuring devices sensitive to transmission variations, and the imaginary part is sensed by index setups monitoring the signal phase changes. Plugging Eqs (4), (5) and (7) into Eq. (8), using the fact that operator $\hat{L}_{s}$ and its adjoint operator $\hat{L}_{s}^{+}\left(0.5 \xi_{s}-i \hat{\kappa}_{s}\right)$ commute with each other and changing $\partial / \partial t$ into $-\partial / \partial t$ by integration-by-parts, we write the output as follows: 


$$
\left.I\left(\tau_{o}\right)=\int_{z_{o}}^{L} d z \int_{-\infty}^{\infty} d t\left[e^{(L-z) \hat{L}_{s}^{+}+L \hat{L}_{s}} E_{s}(0, t)\right]\right]^{*} e^{z \hat{s}_{s}} E_{s}(0, t) \cdot \int_{-\infty}^{\infty} d t^{\prime} \cdot h\left(t+\tau_{z}-t^{\prime}\right)\left|e^{(L-z) \hat{L}_{p}} E_{p}\left(L, t^{\prime}\right)\right|^{2} .
$$

The output $I\left(\tau_{o}\right)$ is similar in form to that of the co-propagating configuration [10] except that the expression here reflects the particular geometry of the counterpropagating pump-and-probe experiments. All three features in the counter-propagating configuration appear in Eq. 9, analytically. The lower bound $z_{o}$ of the integral on $z$ includes the fact that the location where control and signal pulses meet depends of on the initial time delay $\tau_{n}$. $\tau_{z}$, contained in the response function $h$ of the SOA, reflects that the time delay between control and signal pulses is a function of the location $z$. Finally, the fact that control pulse enters the SOA at the opposite side to the signal pulse is indicated in the control pulse evolution, $e^{(L-z) \hat{L}_{p}} E_{p}\left(L, t^{\prime}\right)$.

Similarly to co-propagation pump-and-probe configuration, the effects of the gain slope and pulse walk-off can be treated by expanding the linear operators to their first order of $\partial / \partial t$, i.e. $\hat{\xi}_{j}=\xi_{j}+i \xi_{j}^{\prime} \frac{\partial}{\partial t}, \hat{\kappa}_{j}=\kappa_{j}+i \kappa_{j}^{\prime} \frac{\partial}{\partial t} ; j=p, s$. After a few changes of variables, integration-by-parts', and using the equality $\exp (a \partial / \partial t) f(t)=f(t+a)$ [10], we cast Eq. (9) into the form:

$$
\begin{aligned}
I\left(\tau_{o}\right)= & e^{L \xi_{s}} \int_{z_{o}}^{L} d z \cdot e^{(L-z) \xi_{p}} \int_{-\infty}^{\infty} d t \cdot E_{s}^{*}\left(0, t+\frac{i}{2} \xi_{s}^{\prime}(2 L-z)\right) E_{s}\left(0, t+\frac{i}{2} \xi_{s}^{\prime} z\right) \\
& \cdot \int_{-\infty}^{\infty} d t^{\prime} \cdot h\left(t+\tau_{o}+\frac{z}{v_{p}}+\frac{z}{v_{s}}-t^{\prime}\right) E_{p}\left(L, t^{\prime}+\frac{i}{2} \xi_{p}^{\prime}(L-z)-\Delta k_{z}^{\prime}\right)^{2},
\end{aligned}
$$

where $\Delta k_{z}^{\prime}=\kappa_{p}^{\prime}(L-z)-\kappa_{s}^{\prime} z \cdot \xi_{j}, \kappa_{j}, \xi_{j}^{\prime}$, and $\kappa_{j}^{\prime}(j=p, s)$ are the net gain, the wave vector and their derivatives with respect to angular frequency evaluated at $\omega_{0}$. For specific input control and signal fields, the equation above can be simplified by evaluating the gain, the wave vector, and their first derivatives. In the most experimental setups, the input control and signal pulses assume following waveforms:

$$
\begin{aligned}
& E_{p}(L, t)=\left[A_{p}(t)\right]^{1+i \beta} ; \\
& E_{s}(0, t)=\left[A_{s}(t)\right]^{1+i \beta} .
\end{aligned}
$$

$A_{j}(t)$ with $j=p, s$ are either Sech functions, for pulses derived from a passively mode locked laser, or Gaussian functions for pulses obtained from an actively mode locked laser. Here, $\beta$ is the chirp parameter of the input control and signal pulses.

After several changes of variables and integration-by-parts', similar to the copropagating configuration [10], Eq. (10) becomes more transparent with the input control and signal pulses of the form described by Eqs. (11) and (12): 


$$
\begin{aligned}
I\left(\tau_{o}\right)= & e^{L \xi_{s}} \int_{z_{o}}^{L} d z \cdot e^{(L-z) \xi_{p}} \int_{-\infty}^{\infty} d t \cdot\left\{1+\left[\frac{i \xi_{s}^{\prime}(L-z)}{2}+\frac{i \beta \xi_{s}^{\prime} L}{2}-\frac{i \xi_{p}^{\prime}(L-z)}{2}-\Delta k_{z}^{\prime}\right] \frac{\partial}{\partial t}\right\} \\
& \cdot h\left(t+\tau_{o}+\frac{z}{v_{p}}+\frac{z}{v_{s}}\right) \cdot \int_{-\infty}^{\infty} d t^{\prime} \cdot\left[A_{p}\left(t^{\prime}\right)\right]^{2}\left[A_{p}\left(t^{\prime}+t-\tau_{o}\right)\right]^{2} .
\end{aligned}
$$

The equation becomes more familiar when the cross-correlation function $G^{(2)}(t)$ between control and signal pulses and an impulse response $H(t)$ are defined:

$$
\begin{gathered}
I\left(\tau_{o}\right)=\int_{-\infty}^{\infty} d t \cdot H\left(\tau_{o}-t\right) \cdot G^{(2)}(t) . \\
G^{2}(t)=\int_{-\infty}^{\infty} d t^{\prime} \cdot\left[A_{p}\left(t^{\prime}\right)\right]^{2}\left[A_{s}\left(t^{\prime}+t\right)\right]^{2} . \\
H(t)=e^{L \xi_{s}} \int_{z_{o}}^{L} d z \cdot e^{(L-z) \xi_{p}}\left\{1+\left[\frac{i \xi_{s}^{\prime}(L-z)}{2}+\frac{i \beta \xi_{s}^{\prime} L}{2}-\frac{i \xi_{p}^{\prime}(L-z)}{2}-\Delta k_{z}^{\prime}\right] \frac{\partial}{\partial t}\right\} \\
\cdot h\left(t+\frac{z}{v_{p}}+\frac{z}{v_{s}}\right)
\end{gathered}
$$

Here, the cross-correlation function is even and a few simple transformations are used. The cross-correlation function $G^{(2)}(t)$ becomes the auto-correlation of the laser pulses for control and signal pulses obtained from the same source. It is apparent from Eq. (14), the $H(t)$ is the optical impulse response of the SOA in counter-propagating pump-and-probe experiments.

In conclusion, for a first order perturbation approximation, we have obtained an analytical optical impulse response of the SOA in counter-propagating pump-and-probe experiments. All three different features of the counter-propagating pump-and-probe configuration are taken into account analytically and are embedded into the optical impulse response Eq. (16). The results are applicable to any zinc blende semiconductor active waveguides having a central-symmetry. Higher order accuracy of the optical impulse response can be achieved by including control pulse saturation and self-phase modulation.

\section{References}

[1] I. Glesk, J. P. Sokoloff, and P. R. Prucnal, Electron. Lett., 30, 339 (1994).

[2] Y. H. Kao, I. V. Goltser, M. N. Islam, G, Raybon, CLEO'97 Technical Digest, 94 (1997).

[3] K. L. Deng, I. Glesk, K. I. Kang, and P. R. Prucnal, IEEE Photon. Technol. Lett., 9, 830 (1997).

[4] I. D. Phillips, P. N. Kean, M. J. Doran, I. Bennion, D. A. Pattison, A. D. Ellis, OFC'97 Technical Digest, 273 (1997). 
[5] P. S. Cho, D. Mahgerefteh, J. Goldhar, G. L. Burdge, IEEE Photon. Technol. Lett., 10, 66 (1998).

[6] X. Pan, J. M. Wiesenfeld, J. S. Perino, T. L. Koch, G. Raybon, U. Koren, M. Chien, M.

Young, Miller and C. A. Burrus, IEEE Photon. Technol. Lett., 7, 995 (1995).

[7] A. E. Willner, D. Norte, and E. Park, LEOS'95 Proceedings, 1, 214 (1995).

[8] D. Norte and A. E. Willner, IEEE Photon. Technol. Lett., 8, 712 (19956).

[9] N. S. Patel, K.L. Hall, and K. A. Rauschenbach, Opt. Lett.,, 21, 1466 (1996).

[10] J. Mork, and A. Mecozzi, J. Opt. Soc. Am. B., 13, 1803 (1996).

[11] K. L. Hall, G. Lenz, A. M. Darwish, E. P. Ippen, Opt. Commun., 111, 589 (1994).

[12] K. I. Kang, T. G. Chang, I. Glesk, and P. R. Prucnal, Appl. Opt., 35, 1485 (1996).

[13] D. Zhou, K. I. Kang, I. Glesk, P. R. Prucnal, IEEE LEOS'98, Tech. Digest, WG4 (P.198), Orlando, Florda, Dec. 1998.

[14] K. I. Kang, I. Glesk, and P. R. Prucnal, International Journal of High Speed Electronics and Systems, 7 (1), 125, 1996.

[15] D. Zhou, K. I. Kang, I. Glesk, P. R. Prucnal, IEEE LEOS'98, Tech. Digest, TuA5 (P.16), Orlando, Florda, Dec. 1998.

[16] J. Singh, Semiconductor Optoelectronics-Physics and Technology (McGraw-Hill, New York, 1995) p. 6.

[17] Z. Vardeny, J. Tauc, Opt. Commun., 39 (6), 396 (1981). 